\title{
Performance Assessment of MANET Routing Protocols
}

\author{
Bharath Chandra Mummadisetty, Astha Puri, Shahram Latifi \\ Department of Electrical \& Computer Engineering, University of Nevada, Las Vegas, USA \\ Email:mummadis@unlv.nevada.edu,puri@unlv.nevada.edu,shahram.latifi@unlv.edu
}

Received 29 May 2015; accepted 15 November 2015; published 18 November 2015

Copyright (C) 2015 by authors and Scientific Research Publishing Inc.

This work is licensed under the Creative Commons Attribution International License (CC BY). http://creativecommons.org/licenses/by/4.0/

(c) (i) Open Access

\begin{abstract}
Mobile ad hoc networks use many different routing protocols to route data packets among nodes. Various routing protocols have been developed, and their usage depends on the application and network architecture. This study examined several different routing protocols, and evaluated the performance of three: the Ad Hoc On-Demand Distance Vector Protocol (AODV), the DestinationSequenced Distance-Vector Routing (DSDV), and the Dynamic Source Routing (DSR). These three protocols were evaluated on a network with nodes ranging from 50 to 300, using performance metrics such as average delay, jitter, normal overhead, packet delivery ratio, and throughput. These performance metrics were measured by changing various parameters of the network: queue length, speed, and the number of source nodes. AODV performed well in high mobility and high density scenarios, whereas DSDV performed well when mobility and the node density were low. DSR performed well in low-mobility scenarios. All the simulations were performed in NS2 simulator.
\end{abstract}

Keywords

AODV, DSDV, DSR, Wireless Sensor Networks, MANETs

\section{Introduction}

Mobile ad hoc networks (MANETs) are systems that have ability to self-organize and create temporary networks. They do not require an existing communication setup to interconnect with people and devices. Traditionally, MANETs were used in military applications to improve combat-zone communications as these operations could not rely on a fixed communication infrastructure. Radio signals are prone to interference, and they seldom propagate beyond the line of sight at a radio frequency of 100 MHZ. MANETs can take care of these issues without a pre-existing infrastructure beyond the line of sight [1]. An ad hoc network creates a suitable frame- 
work to address these issues by providing a multi-hop wireless network without pre-placed infrastructure and connectivity beyond the line of sight.

In MANETs, because the nodes move arbitrarily, the network topology can change dynamically and very frequently; this results in route changes with a good chance of packet dropping. Each node acts as a router and generates data independently; sometimes fault detection is difficult since the network management has to be distributed across different nodes. Each node operates at different frequency bands with one or more radio interfaces, which causes asymmetric links [2]. Also, each node might have different processing capabilities due to differences in software/hardware configuration [3]. It is complex to design routing protocols for such networks that change dynamically.

Scalability is critical to the successful deployment of these networks. The steps toward a large network consisting of nodes with limited resources are not straightforward, and present many challenges that still need to be solved in such areas as addressing, routing, location management, configuration management, interoperability, security, and high capacity wireless technologies [4].

\section{Related Work}

Enormous amounts of surveys, studies, and research have been performed to evaluate the performance of various routing protocols for diverse types of network and mobility configurations. This section presents the most relevant studies in this area.

Sujata Agrawal et al. [5] presented a comparative evaluation of ad hoc routing protocols in one-hop and two-hop scenarios, including ad hoc On-Demand Distance Vector (AODV) routing, dynamic source routing (DSR), and ad hocon-demand multipath distance vector (AOMDV) routing.

Humaira Ehsan et al. [6] compared four main ad hocrouting protocols namely DSR, AODV, temporally ordered routing algorithm (TORA), and destination-sequenced distance-vector (DSDV) routing. The first three are on-demand routing protocols that use different routing mechanisms and DSDV is a table-driven protocol. According to their study, DSR outperformed all other protocols in various scenarios and for all tested performance metrics.

Radwan et al. [7] evaluated the performance of three routing protocols for MANET-AODV, DSR, and location-aided routing (LAR)-using the Global Mobile Information System Simulator (GloMoSim). Several performance metrics-normalized routing overhead, average end-to-end delay, throughput, collisions, data packets sent, data packets received, data packets retransmitted, and energy consumption-were compared in detailed. They showed that LAR performed well for normalized routing overhead, AODV for collisions and DSR gave good performance for packets retransmitted.

Azzedine Boukerche et al. [8] compared the performance of several protocols, namely AODV, preemptive AODV (PAODV), Cluster Based Routing Protocol (CBRP), DSR, and DSDV. This study simulated a variety of workload and scenarios, as characterized by mobility, load, and size of the ad hoc network.

Samir R. Das et al. [9] did a comprehensive performance evaluation of routing protocols for the mobile ad hoc networks Sender Policy Framework (SPF), DSDV, TORA, DSR and AODV. They concluded that the multipath protocol, TORA, did not perform well in spite of maintaining multiple redundant path.

Dwivedi et al. [10] performed a study on three protocols, AODV, DSDV, and TORA. According to the study, AODV performed better in MANETs and gave better output; however, the performance of TORA was very poor and was not reliable for MANETs. For wireless sensor networks, four protocols-AODV, DSDV, TORA, and the Low Energy Adaptive Clustering Hierarchy (LEACH)-were selected and evaluated.

Samyak Shah et al. [11] dealt with a simulation model with medium-access control (MAC), and used physical layer models to study interlayer interactions and their performance implications with AODV, DSR and DSDV.

Saiful Azad et al. [12] studied the performance of four wireless routing protocols-AODV, DSR, optimized link state routing (OLSR), and the zone routing protocol (ZRP) for a mobile Worldwide Interoperability for Microwave Access (WiMAX) environment. They concluded that ZRP and AODV protocols outperformed DSR and OLSR.

Rajeshwar Singh et al. [13] dealt with DSR and DSDV routing protocols. For their experiment, a 500-m $\times$ 500-m terrain area was used to illustrate the performance in terms of packet delivery fraction and throughput. The simulation was performed with a network simulator version 2 (NS-2), and the results showed that DSR performed better in both packet delivery fraction and throughput over a DSDV routing protocol. 
Pedro A. Lopez-Fernandez et al. [14] used GloMoSim in their research to investigate various performance statistics and to draw comparisons among different MANET routing protocols, namely AODV, LAR (augmenting DSR), Fisheye State Routing (FSR), wireless routing protocol (WRP), and the Bellman-Ford algorithm.

Samir R. Das et al. [15] performed a study of two prominent on-demand routing protocols for mobile ad hoc networks, namely, DSR and AODV.

S Ahmed et al. [16] analyzed performance differentials to compare three routing protocols, DSR, AODV and TORA. They analyzed the performance over varying loads for each of these protocols using OPNET Modeler 10.5 (OPNET Technologies, Inc).

The following sections discuss the various routing protocols as well as simulation and performance assessment of AODV, DSDV, and DSR routing protocols.

\section{Routing Protocols}

MANET routing protocols can be categorized into three categories: reactive, proactive, and hybrid.

\subsection{Reactive Protocols}

The main characteristic of reactive protocols is that they set up the routes on-demand. When a node wants to start communication with a node to which it does not have any route, the routing protocol will try to establish such a route. The following are the examples of reactive protocols.

\subsubsection{AODV}

Ad Hoc On-Demand Distance Vector Protocol is a reactive or on-demand routing protocol since the routes are established and maintained only when required. It permits the users to find and maintain routes to other users in the network. The routing decisions are made based on distance vectors, i.e., distances measured in hops to all available routers [17]. The protocol supports unicast, broadcast, and multicast communications. The method of traditional routing tables is used, which includes one entry per destination as a mechanism to maintain routing information. The freshness of the routing information is determined by the sequence numbers, which are maintained at each destination. This is done to avoid routing loops. These sequence numbers are carried by all the routing packets [11].

The time stamp is saved in the sequence number, and routes to the destination are saved in the routing table. Each node maintains a sequence number and a routing table. The freshness of the route is determined with the help of sequence numbers: the higher the number, the fresher is the route. This allows discarding the older number.

The routing table consists of a number of entries. Each entry in the table contains the address of the next hop (next node to the destination), a hop count (number of hops to the destination), and a destination sequence number. The variety in data traffic and any kind of mobility can be dealt by AODV. Each route that is active is linked with a time that is stored in the table. Once this time has elapsed, route timeout is triggered and the route is marked as invalid and eventually removed.

Various advantages of AODV protocol include that it supports both unicast and multicast packet transmissions, even for nodes in constant movement. Since it is adaptable to highly dynamic networks, it can respond instantly to the topological changes that affect the active routes. Also, as AODV is a self-starting and loop-free protocol, it does not need any central administrative system to handle the routing process [17].

\subsubsection{DSR}

Dynamic Source Routing is one of the reactive routing protocols. The main distinguishing feature of DSR protocol is that it uses a source routing technique, instead of an independent hop-by-hop technique, in which routing decisions are made by each node. In DSR, the complete hop-by-hop route to the destination is known to the sender. In this protocol, if the node does not have the route that is required in its routing table, nodes in DSR create a route request on an on-demand basis. In source routing, the packet that is going to be routed through the network carries the complete ordered list of nodes in its header through which the packet will pass [18].

The basic operation of DSR is divided into two mechanisms: Route Discovery and Route Maintenance. When a source node $(S)$ has a data packet to be transmitted to the destination node (D), it checks its routing table to find the available route to destination D. If no route is found, the source node activates the route discovery me- 
thod in order to find a route to the destination node. This broadcasts a route request message, which is received by all nodes within the transmission range of the requester. The route request message carries the ID of the source node, destination node, and a list of all intermediate nodes from where the request message has passed.

After the route is established, the source starts sending the data by this route between the source and the destination. Acknowledgement of data packets is achieved hop by hop through the routes. In the case where no acknowledgement is received by any node, the node retransmits the packet until acknowledgement is received or until the maximum number of retransmissions is reached. If no acknowledgement is received and the maximum number of retransmissions is reached, the node considers the route to be broken. The source is informed by the node about the route breakage by sending an error message.

The advantages of DSR protocol are that it allows multiple routes to any destination and allows each sender to select and control the routes used in routing its packets. A node processes a route request packet only if it has not already seen the packet and its address is not present in the route record of the packet. This minimizes the number of route requests propagated in the network. Also, the on-demand feature of DSR reduces the use of bandwidth, especially in cases where mobility is low [19].

\subsubsection{ACOR}

Admission Control enabled On-demand Routing protocol enables quality of service (QOS) support. In ACOR, a route that requires QOS is created on demand without the need to maintain routing information and exchange the routing table periodically. When a route is required by the source node, a Route Request packet is broadcasted by the source node towards the destination. Once the destination is achieved, it responds by unicasting a Route Reply packet to the source node. ACOR is based on simple and efficient techniques to provide quality of service.

A QOS parameter is represented by a local cost function at each node. The route request packet is received at each node. The requested resources are implicitly reserved, and, after the local cost function is appended to the global function. The global function will be accumulated along the route from the source to the destination to represent the end-to-end route quality. After this step, the value of the global cost function is recorded and sent back in the Route Reply packet towards the source node. Once the source node receives the route reply packet, based on the value of the global cost function, the source node will choose the route [20].

\subsubsection{ABR}

Associativity-Based Routing protocol does not consistently maintain routing information in every node, and is associativity-based. A route is selected based on nodes having associativity states. Thus, the routes are longlived and do not need to be restarted often, leading to higher attainable throughput. Route requests are broadcast on a per-need basis. When the association property is violated to discover shorter routes, the localized query is incorporated into the protocol; in order to shorten the route recovery time, quick-abort mechanisms are incorporated into the protocol. Additionally, a dynamic scheme for cell-size adjustment is introduced to increase cell capacity and lower transmission power requirements. The protocol is free from packet duplicates, loops, and deadlock, and has scalable memory requirements.

ABR only maintains routes for sources that actually desire routes. However, ABR does not employ route re-construction based on alternate route information, thereby avoiding stale routes. In addition, to avoid packet duplicates, only the best route will be selected and used, while all other possible routes remain passive. The selected route tends to be more long-lived due to the property of associativity [21].

\subsection{Proactive Protocols}

In networks where a proactive routing protocol is used, every node maintains one or more tables that demonstrate the entire topology of the network. There is a need to maintain up-to-date routing information from each node to every other node; thus, the tables are updated regularly. To achieve this, topology information needs to be exchanged between the nodes on a regular basis, leading to high overhead on the network.

\subsubsection{OLSR}

Optimized Link State Routing Protocol is one of the proactive routing protocols; that is, whenever needed, the routes always are available. In order to preserve the bandwidth, message flooding in OLSR is optimized. This 
optimization is based on a technique called Multipoint Relaying, in which the nodes are free to move arbitrarily and can organize themselves randomly. Each mobile host is treated by the node as a router.

In OLSR, the pre-computed route information about all the nodes is contained in each node. This information is exchanged periodically by protocol messages. Hop-by-hop routing is performed by OLSR, in which each node uses its recent topology information for routing. Each node selects a set of its neighbor nodes as Multi Point Relays (MPRs). The control traffic is forwarded only by those nodes that are selected as MPRs. MPRs are selected in a way such that 2-hop neighbors can be reached through at least one MPR node.

By providing the link-state information, this provides the shortest path routes to all the destinations [22]. This information is passed periodically by nodes selected as MPRs as a part of their control messages. Control messages are used to pass the information to neighboring MPRs. The route from the starting node to the destination node is formed by the help of MPRs. The main purpose of selecting MPRs is to diminish flooding overhead and provide optimal flooding distance.

\subsubsection{DSDV}

Destination-Sequenced Distance-Vector Routing is one of the table-driven or proactive routing schemes based on the Bellman-Ford algorithm, developed by C. Perkins and P. Bhagwat in 1994. One of the main contributions of the algorithm was to solve the Routing Loop problem. The routing table consists of various entries, and each entry consists of a sequence number that is even if a link is present; otherwise, it is odd [23]. Routing information is distributed between nodes by sending full dumps occasionally and smaller incremental updates more frequently.

DSDV was one of the early available algorithms suitable for creating ad hoc networks with a small number of nodes. The main feature of DSDV is that it requires its routing tables to be updated regularly; this uses the battery's power and a small amount of bandwidth, even when the network is idle. DSDV is not suitable for highly dynamic networks because whenever there is a change in topology, the connection needs to be re-established. This, in turn, requires a new sequence number to be generated [16].

The transmission of packets from one node to another is accomplished according to the routing table. The routing table, maintained by each node, has an entry for each of the nodes of the network. This protocol guarantees loop-free paths. Instead of maintaining multiple paths to every destination, DSDV maintains only the best path available to the destination, thus reducing the amount of space of the routing table. If any node detects that a particular route to a destination node is broken, then its hop number is set to infinity and the sequence number is updated. This shows that there is freshness of route in this protocol [16].

\subsubsection{CGSR}

In the protocol known as Cluster head Gateway Switch Routing (CGSR), the nodes that are mobile in nature are clustered into groups, and a cluster head is elected for each group. All the nodes in the communication range of the cluster head belong to its cluster. A node that belongs in the communication range of two or more cluster heads is referred as a gateway node.

This cluster head scheme causes performance degradation in a dynamic network. To avoid this, CGSR uses a Least Cluster Change (LCC) algorithm. In this algorithm, if a change in the network causes one of the nodes to move out of range or two cluster heads merge into one, only then will the cluster head change occur.

The CGSR works in the following manner. The packet is transmitted to the cluster head from its source. Then, the packet is sent to the gateway node. The gateway node sends it to the next cluster head, and so on, until the packet reaches the destination cluster head. At that point, the packet is sent to the destination by the destination cluster.

A cluster member table is maintained by each node. This table has mapping from each node to its respective cluster head, and each node regularly updates its table. Additionally, a routing table that determines the next hop to reach the destination cluster is maintained by each node. According to the routing table and cluster member table, when a packet is received, the node finds the cluster head that is nearest along the route. Then, the packet consults the routing table in order to find the next hop in order to reach to the cluster head that was selected earlier. Finally, the packet is transmitted to that node [24].

\section{Methodology}

For simulation purposes in this study, the performance of AODV, DSR, and DSDV protocols were evaluated 
using Average Delay, Control Overhead, Dropping Ratio, Jitter, Normal Overhead, Packet Delivery Ratio, and Throughput. The Ubuntu operating system and NS2 simulator version 2.35 were used. For the protocols being evaluated, scripts were written in tool command language (TCL) to simulate the traffic pattern as well as to create trace files and the network animator. AWK scripts were written to process the trace files in order to calculate the performance metrics of the three protocols.

For the test network that was developed, the size of the nodes varied from 50 to 300, with random movements to simulate a scenario close to reality. Therefore, the nodes moved freely in a random fashion. Nodes $0,1,2,3,4$, 5, 6, 7, 9 were the source nodes, and Node 8 was the sink.

The following command was used to execute the tcl file:

ns $<$ source_file $>$.tcl

To generate the trace file and the. nam file for network animation, the following command was used.

nam <network_animator $>$.nam

To open network animator and provide a simulation of network traffic between nodes, the following command was used.

awk-f $<$ script $>$.awk $<$ tracefile $>$.tr

This command executed AWK scripts to process the trace files.

Figure 1 shows the screenshot of the network animator.

\section{Results and Discussion}

Table 1 provides the configuration used to setup the network.

The simulator used for the study was NS2 version 2.35. The channel type was a wireless channel, and "Channel/Wireless Channel" denotes the base class and sub class. 802.11 standards were used for wireless networks. Priority Queue was used for the Interface queue type, which means that when the packets of that protocol were received, they were given priority. The queue size was assumed to be 50 .

\subsection{Variation in Number of Nodes}

Figure 2 shows the variation of the packet delivery ratio for all the protocols when the number of nodes were varied. AODV had the lowest packet delivery ratio. For the DSDV protocol, the value gradually increased with the increase in number of nodes. DSR was efficient when it had more nodes as well.

As shown in Figure 3, AODV has the least average end-to-end delay. In contrast, the value of DSR gradually increased when the node density increased. Initially, DSDV had a greater average delay than the other two

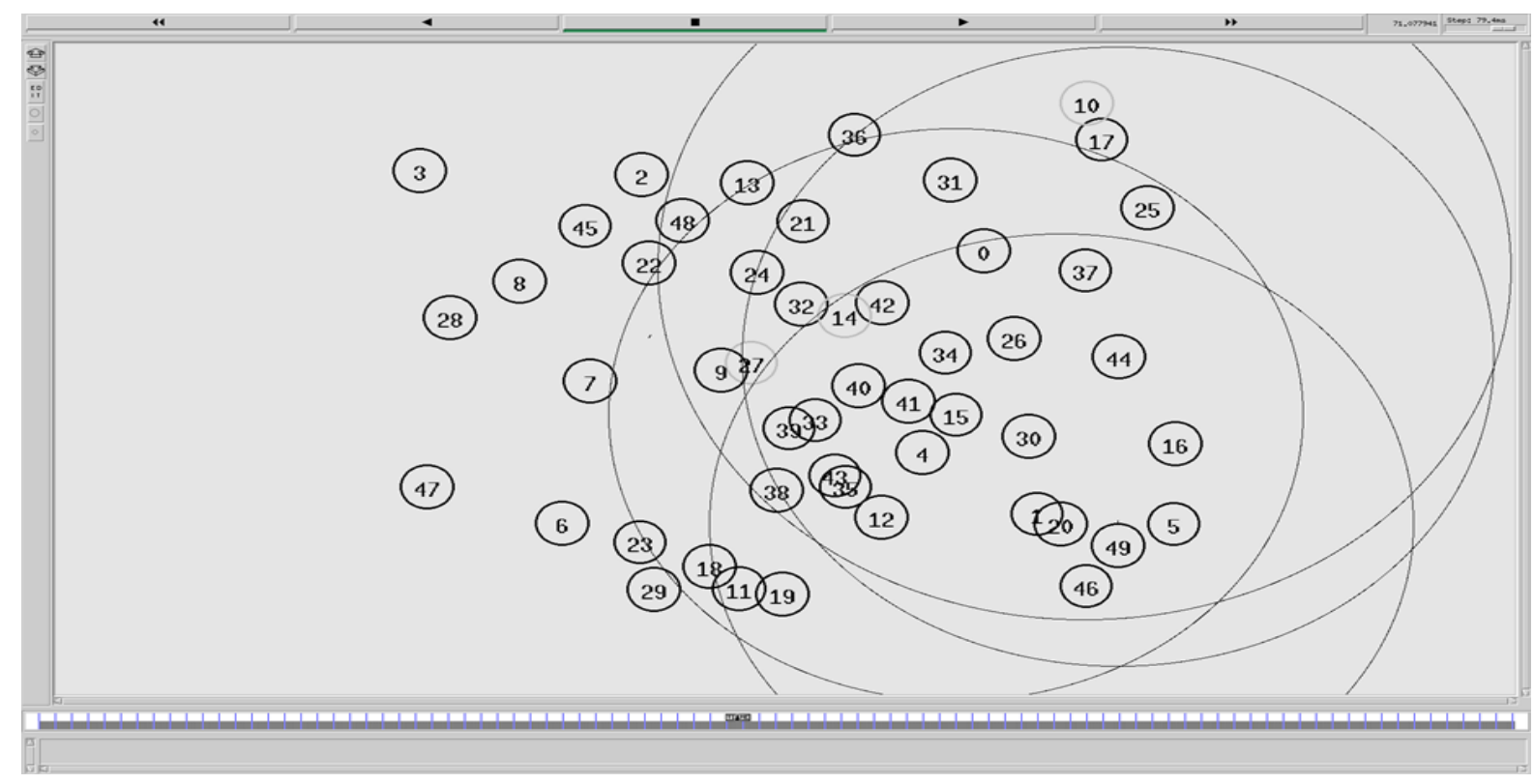

Figure 1. Network animator. 


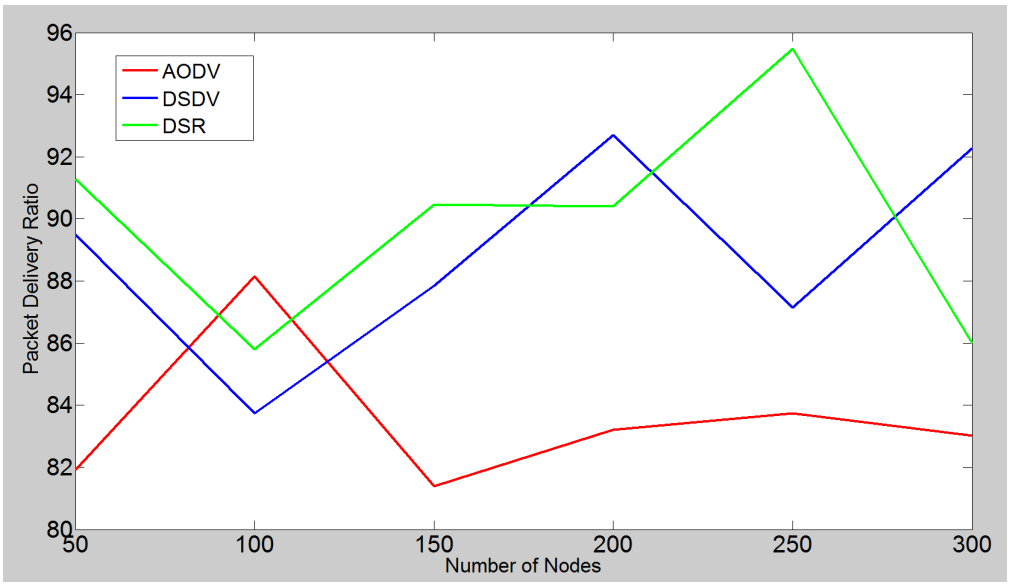

Figure 2. Packet delivery ratio of the AODV, DSDV, and DSR protocols with the variation of the number of nodes.

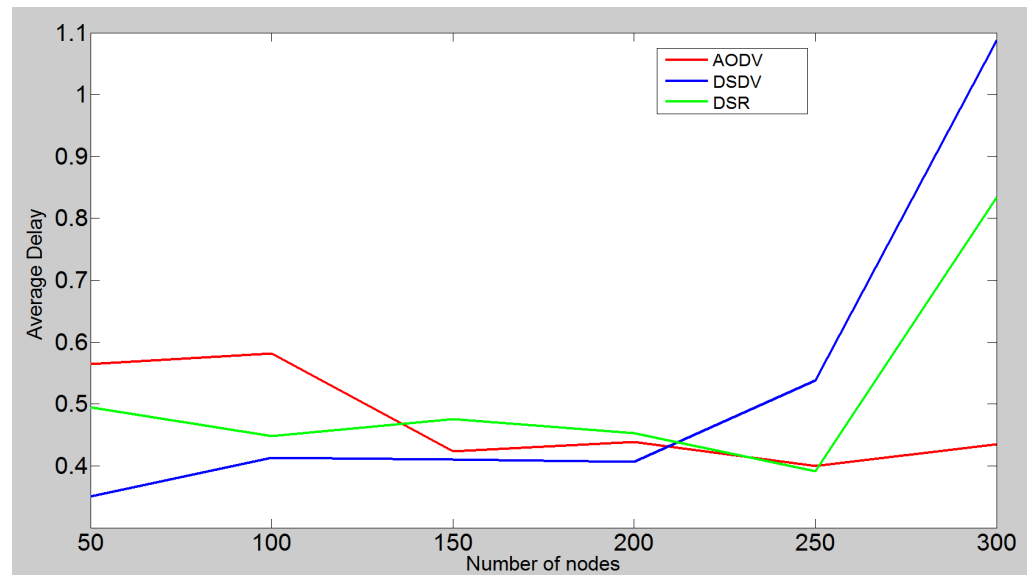

Figure 3. Average end-to-end delay when the number of nodes varied.

Table 1. Parameters used for performance evaluation.

\begin{tabular}{cc}
\hline Parameter & Value \\
Simulator & NS-2 (Version 2.35) \\
Channel Type & Channel/Wireless Channel \\
Network Interface Type & Phy/Wireless Phy \\
Mac Type & Mac/802.11 \\
Interface Queue Type & Queue/Drop Tail/Pri Queue \\
Link Layer Type & LL \\
Antenna & Antenna/Omni Antenna \\
Maximum Packet in ifq & 50 \\
Area & $500 \times 400$ \\
Number of Nodes & 50 to 300 \\
Number of mobile Nodes & 9 \\
Simulation time & 150 sec \\
Source Type & TCP \\
Routing Protocols & AODV, DSR, DSDV \\
\hline
\end{tabular}


protocols, but that delay increased with an increase in the node density.

Regarding the variation in jitter for the protocols (Figure 4), AODV outperformed the other two protocols in terms of jitter as the node density increased. DSDV had high jitter with an increase in node density, and DSR had a moderate amount of jitter.

Variations in the normalized overhead is shown in Figure 5. For DSDV, the normalized overhead overshot as the node density increased; furthermore, its performance was similar to the other two protocols when the node density was lower. There were less variations with AODV and DSR.

Regarding the variation of throughput (Figure 6), AODV outperformed the other protocols in terms of throughput when the node density was high. The throughput of DSDV decreased drastically with an increase in node density. For DSR, the value remained almost constant when the node density was increased.

\subsection{Variation in Node Speed}

The speed of the nodes were varied from $10 \mathrm{~m} / \mathrm{s}$ to $60 \mathrm{~m} / \mathrm{s}$, and the performance metrics were evaluated. Figure 7 shows the variation of the packet delivery ratio for all the three protocols. DSR performed the best when the speed of the nodes was moderate, but the performance of DSDV deterioted. AODV had the least performance, in this case.

As shown in Figure 8, the variation of average delay for AODV was high when compared to the other two protocols. The performance of DSDV was better when the speed of the nodes increased; it was fairly constant throughout, and increased after $50 \mathrm{~m} / \mathrm{s}$. DSR performed well when the speed was moderate.

With regard to the variation of jitter (Figure 9), AODV had the highest jitter when compared to other two protocols.

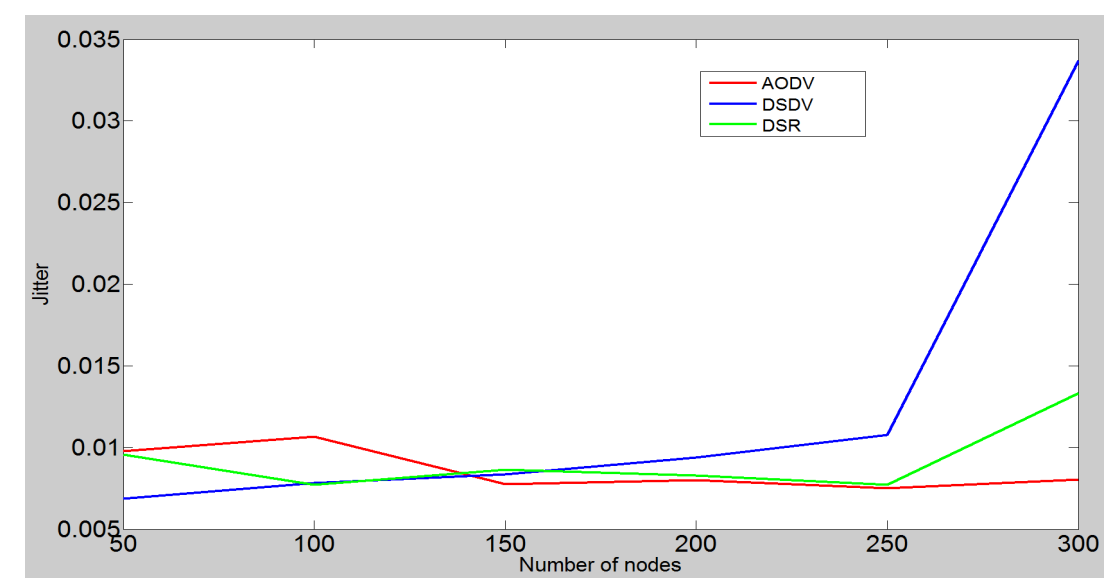

Figure 4. Jitter with the variation in the number of nodes.

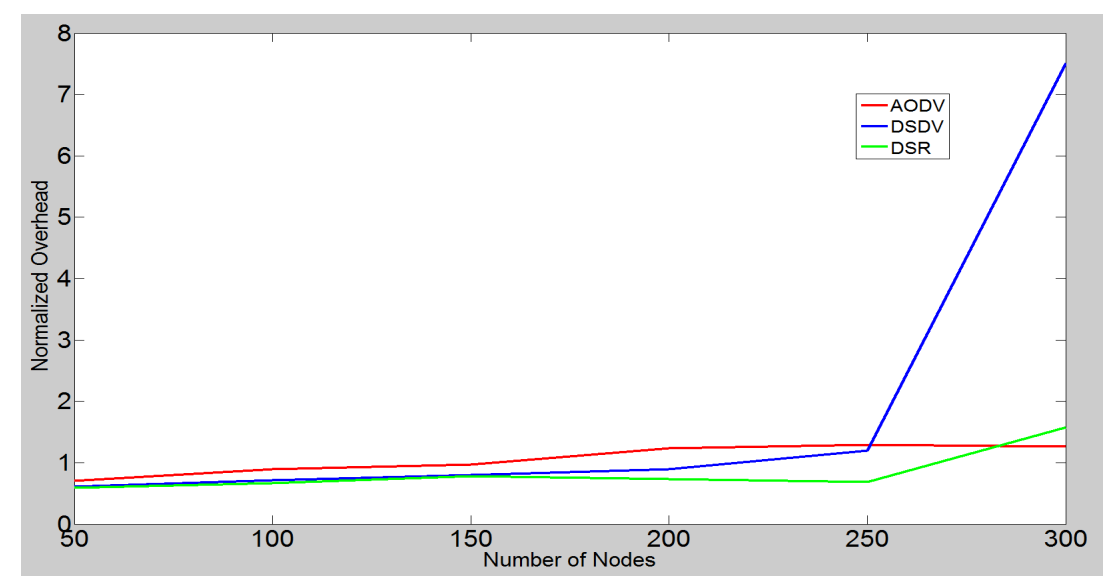

Figure 5. Normalized overhead with the variation in the number of nodes. 


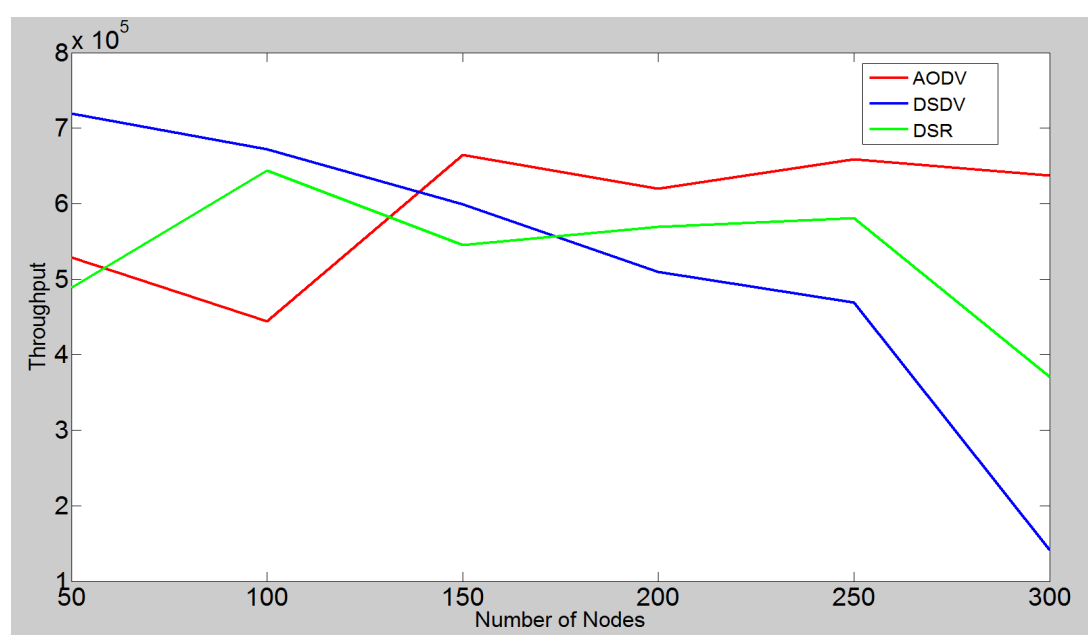

Figure 6. Throughput when the number of nodes varied.

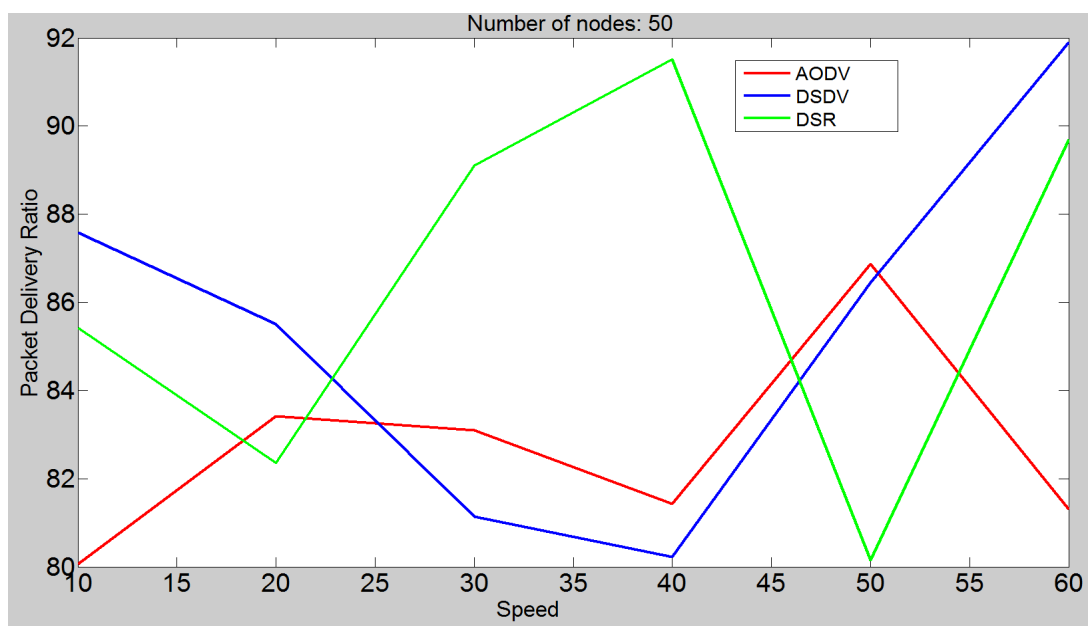

Figure 7. Packet delivery ratio with variation in speed.

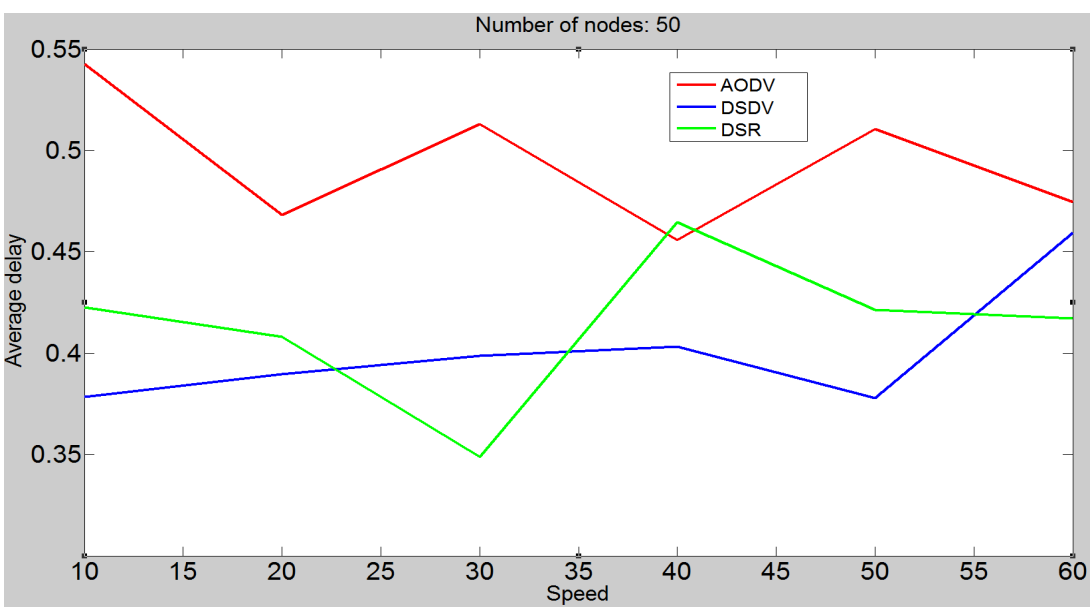

Figure 8. Average delay with variation in speed.

The variation of normalized overhead (Figure 10) of AODV was high. In this case, DSR outperformed the other two protocols. 


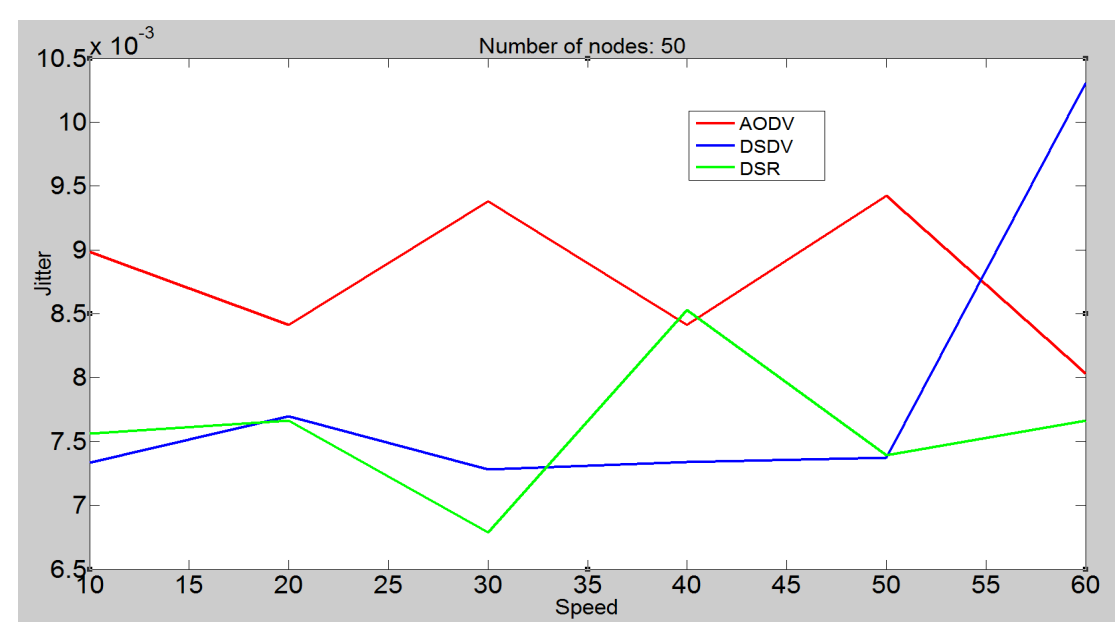

Figure 9. Jitter with variation in speed.

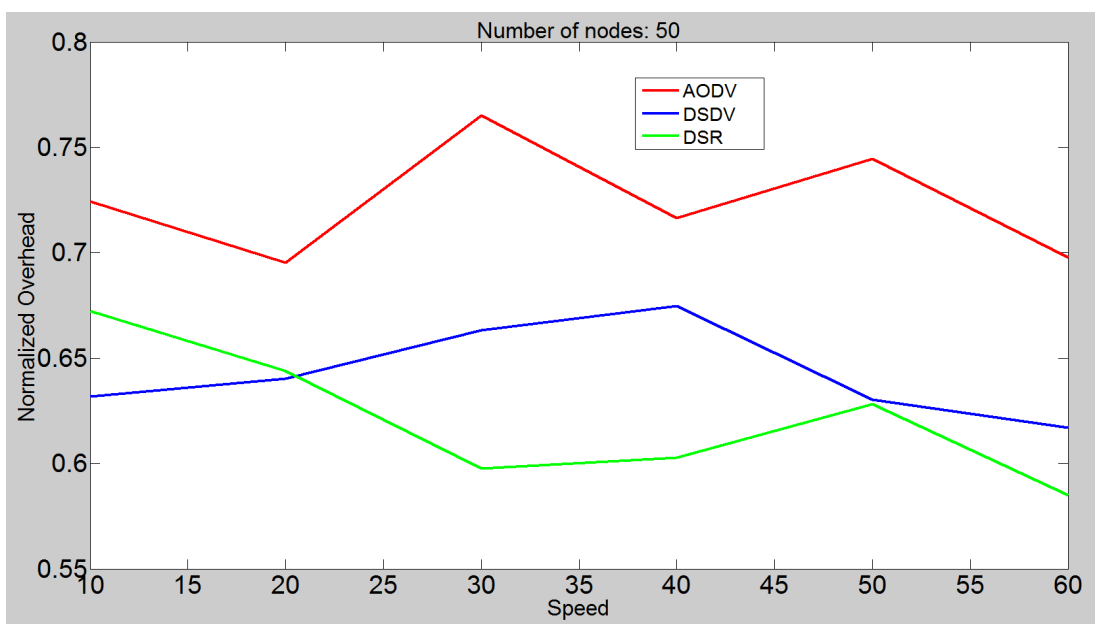

Figure 10. Normalized overhead with variation in speed.

Figure 11 shows the variation of throughput among the three protocols. Throughput of DSDV steadily decreased with an increase in speed. AODV had the least amount of throughput. DSR had a high throughput because it used more than one path to deliver packets from source to destination.

\subsection{Variation in Number of Source Nodes}

The number of source nodes were varied from 10 to 60 , and the performance metrics were analyzed. The sink (destination node) remained Node 8.

Figure 12 shows the variation of packet delivery ratio when the number of source nodes was varied. DSR had a high packet delivery ratio, up to $95 \%$, when number of source nodes varied from 30 to 50 . AODV had a low packet delivery ratio in comparison to the other two protocols.

Variation of normalized overhead is shown in Figure 13. Normalized overhead of DSDV protocol was low and outperformed the other protocols. DSR overshot; with AODV, the normalized overhead gradually increased with the increase in the source-node density.

Figure 14 show the throughput variation among the three protocols. AODV and DSDV performed well, and DSR had the poorest performance.

Regarding the average delay variation (Figure 15), the performance of AODV and DSDV was nearly same, whereas the delay for DSR was high.

Figure 16 shows the variation of jitter among the three protocols. Jitter for DSR was high when the number of source nodes varied; for AODV and DSDV, it was low and nearly same. 


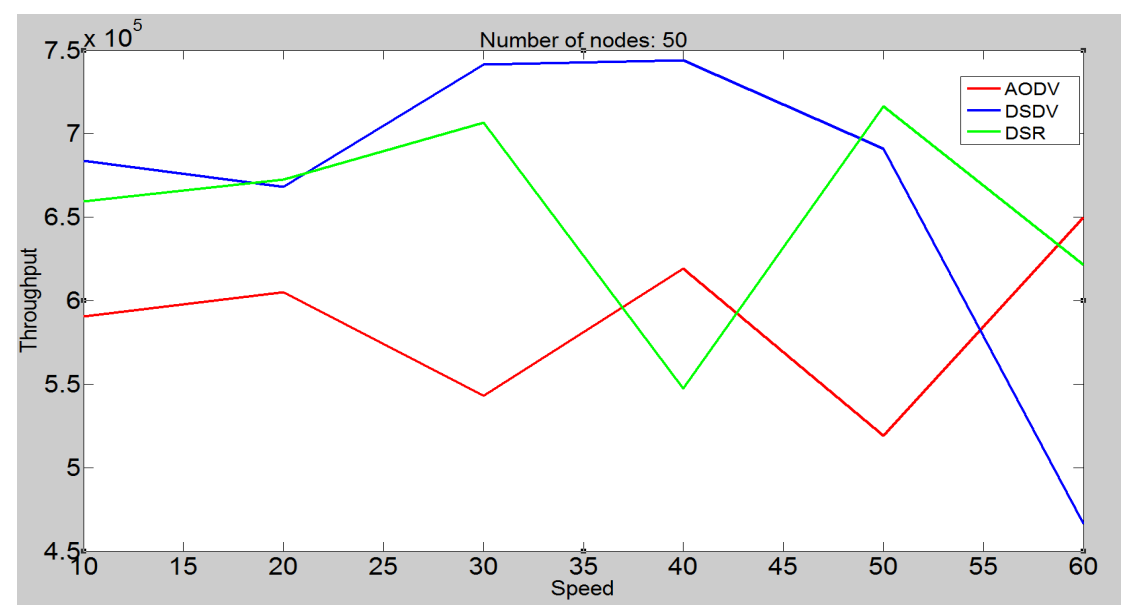

Figure 11. Throughput with variation in speed.

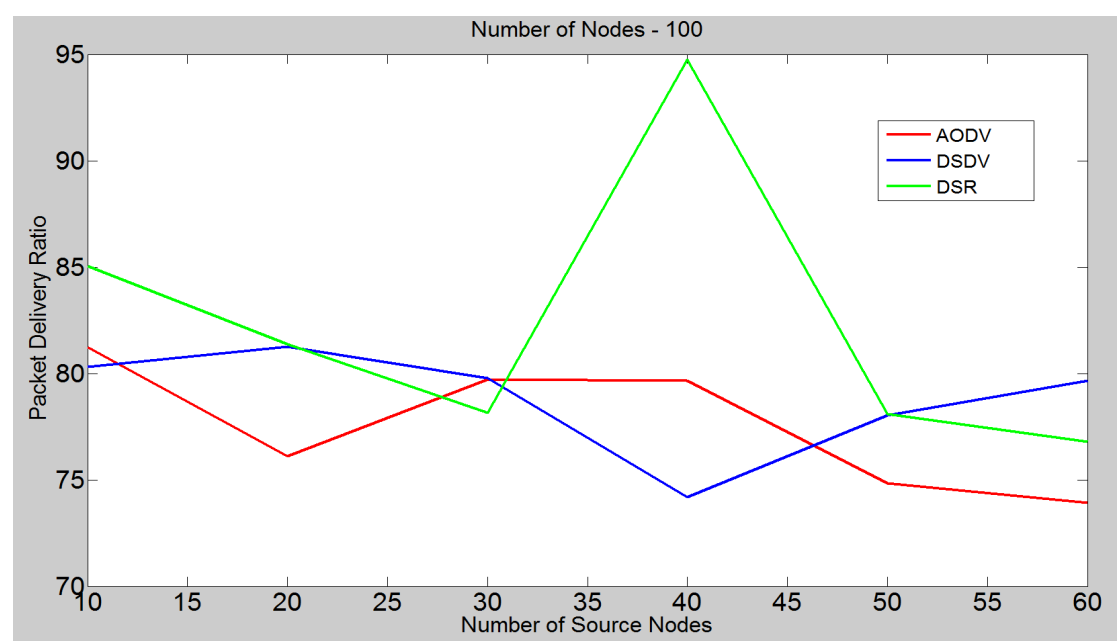

Figure 12. Packet delivery ratio when the number of source nodes varied.

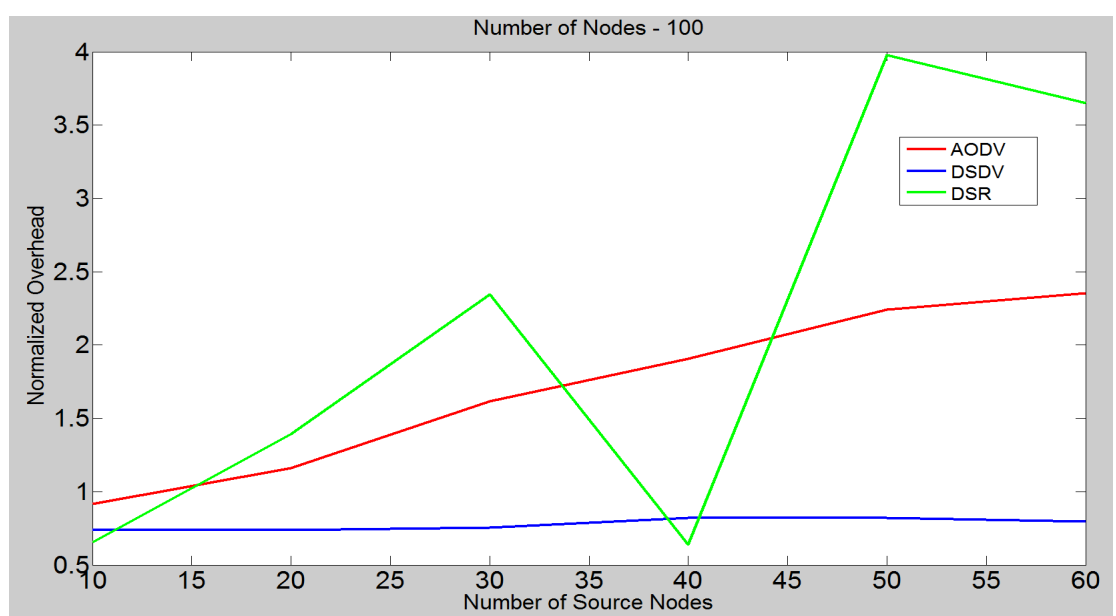

Figure 13. Normalized overhead with the variation in source nodes.

\subsection{Variation in Queue Length}

Figure 17 gives the variation of delay when the queue length was changed. The average delay of DSR was low 


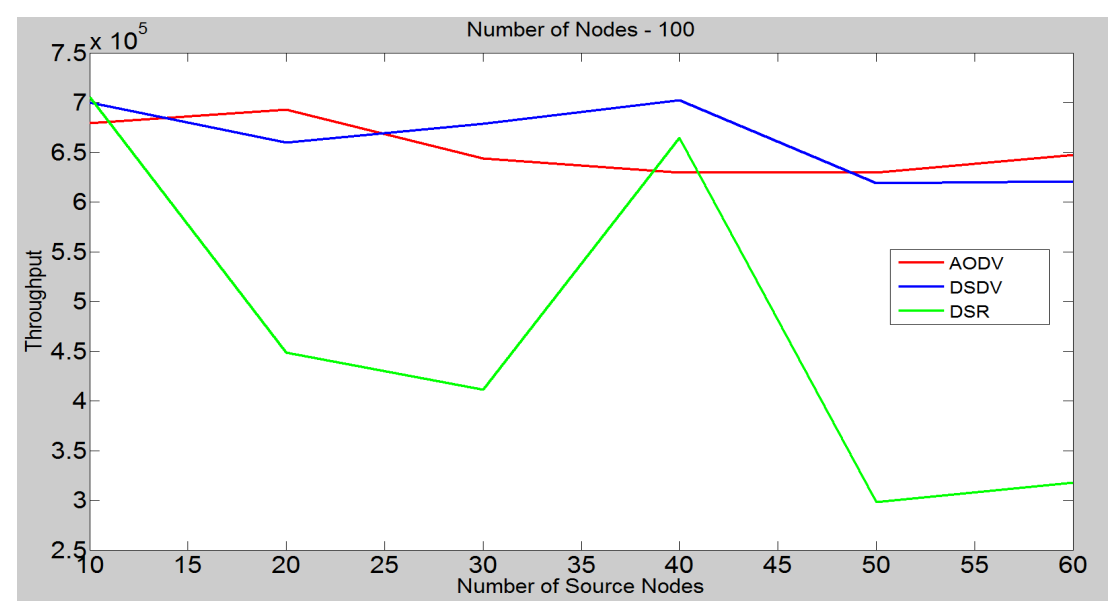

Figure 14. Throughput with the variation in source nodes.

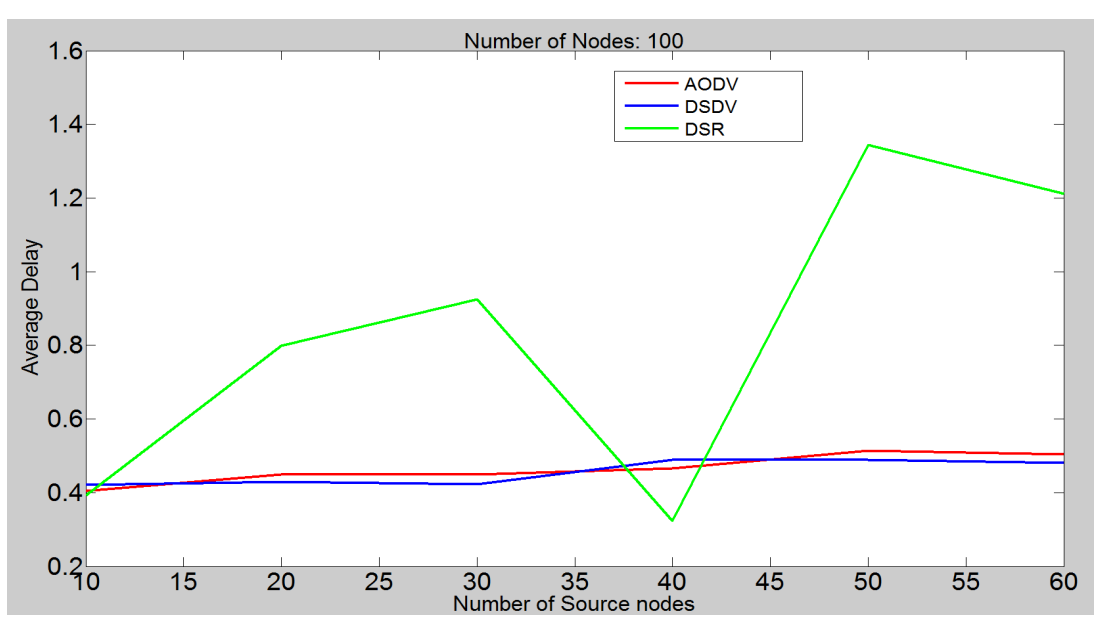

Figure 15. Average delay with the variation in source nodes.

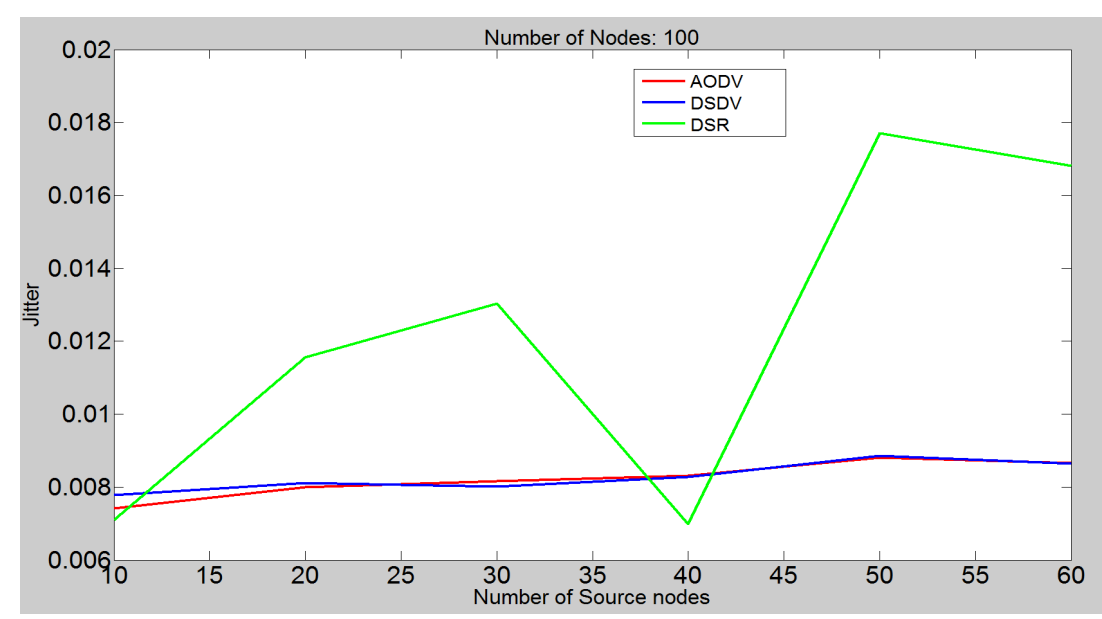

Figure 16. Jitter with the variation in source nodes.

when compared with the other two protocols. The average delay of DSDV seemed to improve with the increase in the number of nodes.

Jitter variation is shown in Figure 18. The jitter for DSDV overshot with an increase in the number of nodes. 
Figure 19 shows the variation of normalized overhead. For DSDV, the value overshoots with the increase in the number of nodes.

Variation in the packet delivery ratio is shown in Figure 20. DSR had a high value initially; however, as the node density increased, its value decreased. AODV had a nearly constant low value. DSDV outperformed the other two protocols.

Throughput variation, shown in Figure 21, decreased steadily for DSDV with the increase in the number of nodes. AODV and DSR did not show stable behavior.

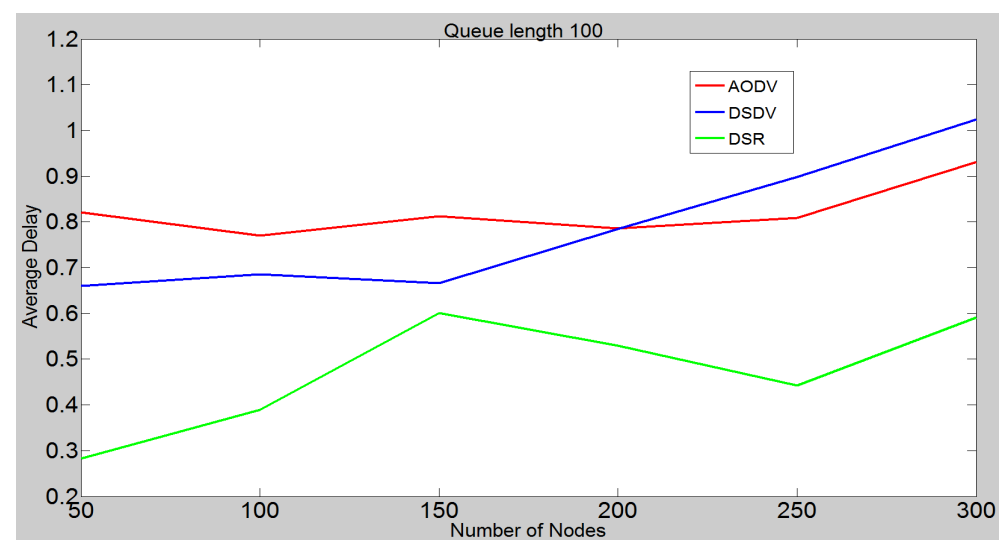

Figure 17. Average delay with the variation in queue length.

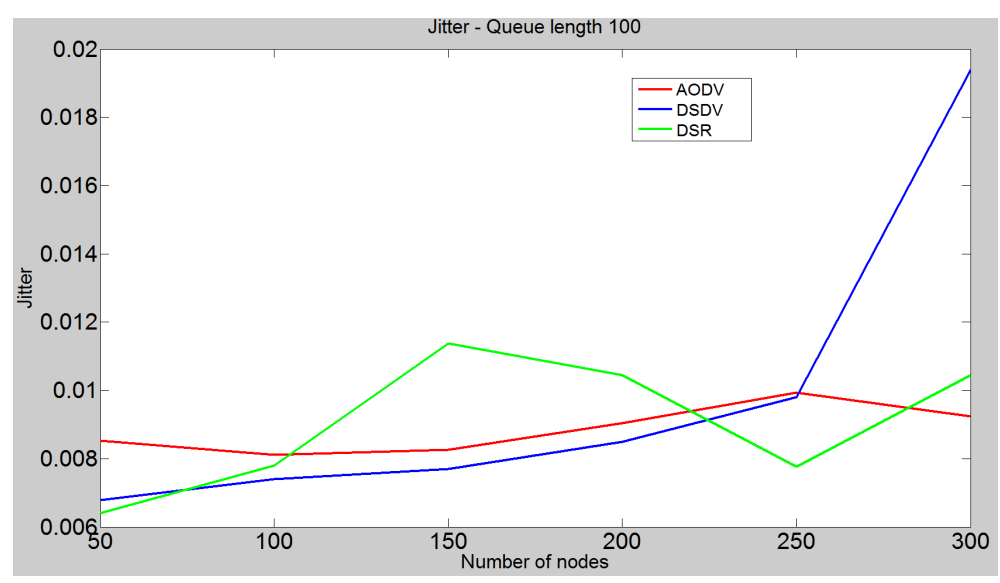

Figure 18. Jitter with the variation in queue length.

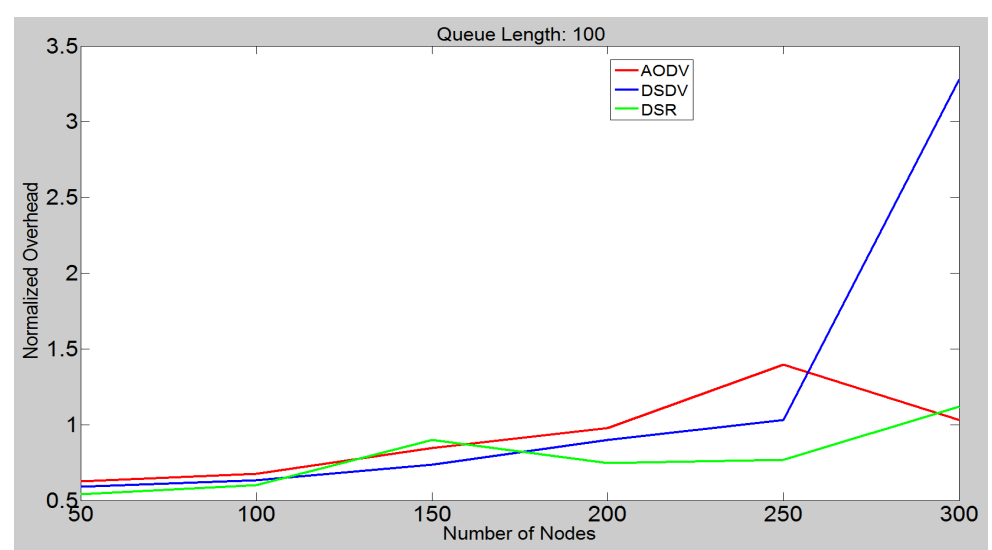

Figure 19. Normalized overhead with the variation in queue length. 


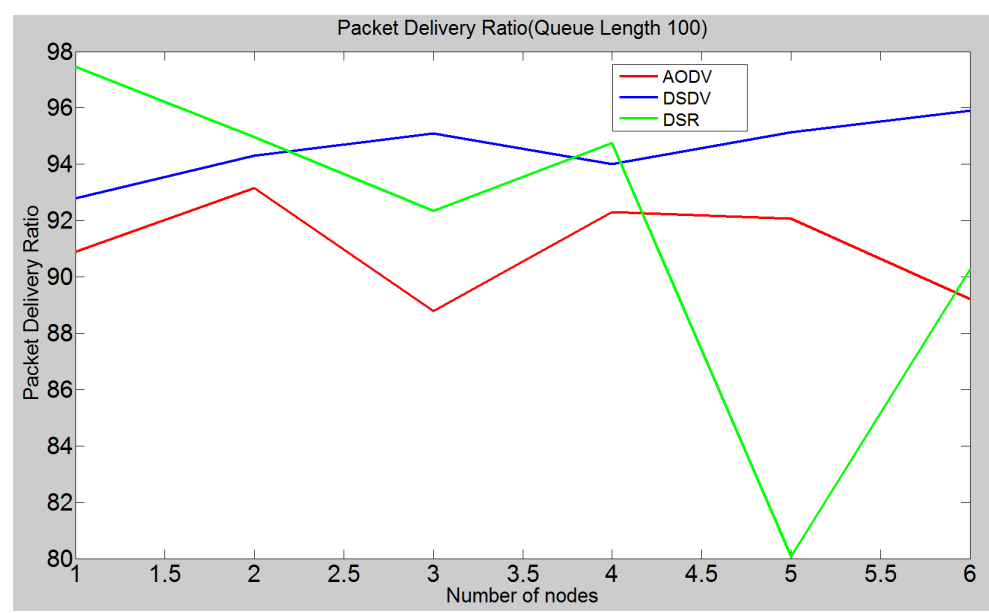

Figure 20. Packet delivery ratio with the variation in queue length.

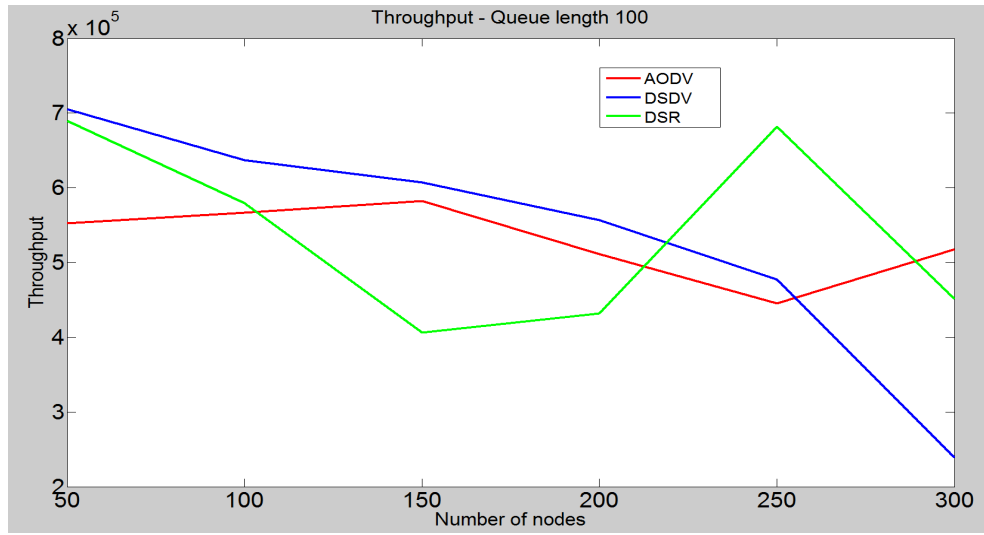

Figure 21. Throughput with the variation in queue length.

\section{Conclusions}

In this study, based on the analysis and simulation results, it was concluded that there was no one best protocol for all the scenarios tested. The choice of protocol depended on the network and parameters considered. It was concluded that AODV performed well in high mobility and high density scenarios, whereas DSDV performed well when mobility and node density were low. DSR performed well for low-mobility scenarios.

Future study may include the simulation of other protocols and the security aspects of the protocols.

\section{Acknowledgements}

This work was supported by the National Science Foundation (NSF) grant \#EPS-IIA-1301726 and in part by the Nevada NASA EPSCOR Space Grant. Any findings, conclusions, or recommendations expressed in the material are those of the author(s), and do not necessarily reflect the views of NSF.

\section{References}

[1] Freebersyser, J.A. and Leiner, B. (2001) A DoD Perspective on Mobile Ad Hoc Networks. Addison Wesley, Reading, 29-51.

[2] Chlamtac, I. and Lerner, A. (1986) Link Allocation in Mobile Radio Networks with Noisy Channel. IEEE INFOCOM, Bar Harbor, April.

[3] Chlamtac, I. and Lerner, A. (1987) Fair Algorithms for Maximal Link Activation in Multi-Hop Radio Networks. IEEE Transactions on Communications COM-35.

[4] Chlamtac, I., Conti, M. and Liu, J.J.N. (2003) Mobile Ad Hoc Networking: Imperatives and Challenges. Ad Hoc Net- 
works, 1, 13-64. http://dx.doi.org/10.1016/S1570-8705(03)00013-1

[5] Agrawal, S., Daigavane, M.B. and Kulat, K.D. (2012) Performance Evaluation of Routing Protocols for Wireless Ad Hoc Network. International Journal of Advanced Engineering Technology, 13-17.

[6] Ehsan, H. and Uzmi, Z.A. (2004) Performance Comparison of Ad Hoc Wireless Network Routing Protocols. 8th International Multitopic Conference, 457-465.

[7] Radwan, A.A.A., Mahmoud, T.M. and Houssein, E.H. (2011) Evaluation Comparison of Some Ad Hoc Networks Routing Protocols. Egyptian Informatics Journal, 12, 95-106. http://dx.doi.org/10.1016/j.eij.2011.04.001

[8] Boukerche, A. (2004) Performance Evaluation of Routing Protocols for Ad Hoc Wireless Networks. Mobile Networks and Applications, 9, 333-342. http://dx.doi.org/10.1023/B:MONE.0000031592.23792.1c

[9] Das, S.R. and Sengupta, R. (1998) Comparative Performance Evaluation of Routing Protocols for Mobile, Ad Hoc Networks. 7th International Conference on Computer Communications and Networks, Lafayette, 12-15 October 1998, 153-161. http://dx.doi.org/10.1109/icccn.1998.998772

[10] Dwivedi, A.K., Kushwaha, S. and Vyas, O.P. (2009) Performance of Routing Protocols for Mobile Ad Hoc and Wireless Sensor Networks: A Comparative Study. International Journal of Recent Trends in Engineering, 2.

[11] Shah, S., Khandre, A., Shirole, M. and Bhole, G. (2008) Performance Evaluation of Ad Hoc Routing Protocols Using NS2 Simulation. Mobile and Pervasive Computing (CoMPC-2008), Chennai, 7-8 August 2008, 167-171.

[12] Azad, S., Uddin, M., Anwar, F. and Rahman, A. (2008) Performance Evaluation of Wireless Routing Protocols in Mobile WiMAX Environment. Proceedings of the International Multi Conference of Engineers and Computer Scientists, 2, 1109-1114.

[13] Singh, R., Singh, D.K. and Kumar, L. (2011) Performance Evaluation of DSR and DSDV Routing Protocols for Wireless Ad Hoc Networks. International Journal of Advanced Networking and Applications, 2, 732-737.

[14] Fernandez, L. and Pedro, A. (2008) Routing Protocol Performance Evaluation for Mobile Ad-Hoc Networks. UNF Thesis and Dissertations, Paper 293.

[15] Das, S.R., Perkins, C.E. and Royer, E.M. (2001) Performance Comparison of Two On-Demand Routing Protocols for Ad Hoc Networks. IEEE Personal Communications, 8, 16-28. http://dx.doi.org/10.1109/98.904895

[16] Usop, N.S.M., Abdullah, A. and Abidin, A.F.A. (2009) Performance Evaluation of AODV, DSDV \& DSR Routing Protocol in Grid Environment. International Journal of Computer Science and Network Security, 9.

[17] Hassnawi, L.A., Ahmad, R.B, Yahya, A., Aljunid, S.A. and Elshaikh, M. (2012) Performance Analysis of Various Routing Protocols for Motorway Surveillance System Cameras’ Network. International Journal of Computer Science Issues, 9, 52-62.

[18] Asar, A. and Akbar, Z. (2009) Evaluation of AODV and DSR Routing Protocols of Wireless Sensor Networks for Monitoring Applications. Thesis, Blekinge Institute of Technology, Department of Electrical Engineering with Emphasis on Telecommunication, Karlskrona.

[19] Johnson, D., Hu, Y. and Maltz, D. (2007) Dynamic Source Routing Protocol (DSR). RFC 4728, Network Working Group.

[20] Kettaf, N. and Abouaissa, H. (2007) Admission Control Enabled on Demand Routing (ACOR).

[21] Toh, C.K. (1997) Associativity-Based Routing for Ad Hoc Mobile Networks. Wireless Personal Communications, 4, 103-139. http://dx.doi.org/10.1023/A:1008812928561

[22] Mostafavi, A., Moghanjoughi, A.A. and Mousavi, H. (2012) A Review and Performance Analysis of Reactive and Proactive Routing Protocols on MANET. Network and Communication Technologies, 1, 48-58.

[23] Perkins, C.E. and Bhagwat, P. (1994) Highly Dynamic Destination-Sequenced Distance-Vector Routing (DSDV) for Mobile Computers. ACM SIGCOMM Computer Communication Review, 24, 234-244. http://dx.doi.org/10.1145/190314.190336

[24] Misra, P. (1999) Routing Protocols for Ad Hoc Mobile Wireless Networks. Courses Notes. http://www.cse.wustl.edu/ jain/cis788-99/ftp/adhoc_routing/ 